\title{
Corrigendum: Defeat, Entrapment, and Positive Future Thinking: Examining Key Theoretical Predictors of Suicidal Ideation Among Adolescents
}

\author{
Olivia H. Pollak, Eleonora M. Guzmán, Ki Eun Shin and Christine B. Cha* \\ Department of Clinical and Counseling Psychology, Teachers College, Columbia University, New York, NY, United States
}

Keywords: suicide, defeat, entrapment, future thinking, integrated motivational-volitional model, adolescence

\section{A Corrigendum on}

Defeat, Entrapment, and Positive Future Thinking: Examining Key Theoretical Predictors of Suicidal Ideation Among Adolescents

by Pollak, O. H., Guzmán, E. M., Shin, K. E., and Cha, C. B. (2021). Front. Psychol. 12:590388. doi: $10.3389 /$ fpsyg.2021.590388

In the original article, there were mistakes in the legends for Figures 2 and 3 as published. The figure legends incorrectly stated that greater and lower levels of positive future thinking (Figure

OPEN ACCESS

Edited and reviewed by: Roumen Kirov,

Institute of Neurobiology, Bulgarian Academy of Sciences (BAS), Bulgaria

*Correspondence:

Christine B. Cha

cbc2120@tc.columbia.edu

Specialty section:

This article was submitted to

Psychopathology,

a section of the journal

Frontiers in Psychology

Received: 14 July 2021

Accepted: 16 August 2021

Published: 16 September 2021

Citation:

Pollak OH, Guzmán EM, Shin KE and Cha CB (2021) Corrigendum: Defeat,

Entrapment, and Positive Future Thinking: Examining Key Theoretical Predictors of Suicidal Ideation Among

Adolescents.

Front. Psychol. 12:741504. doi: 10.3389/fpsyg.2021.741504
2) were defined at $-1 \mathrm{SD}$ and $+1 \mathrm{SD}$, respectively; and that more vs. less realistic positive future thinking levels were defined at $+1 \mathrm{SD}$ and $-1 \mathrm{SD}$, respectively (Figure 3 ). In fact, it was the opposite. Additionally, the text of the original article also incorrectly stated that higher and lower levels of positive future thinking, and higher and lower levels of unrealistic positive future thinking, were probed at -1 SD below and +1 SD above the mean, respectively. In fact, it was the opposite: higher positive and higher unrealistic future thinking were probed at $+1 \mathrm{SD}$ above the mean, and lower positive and lower unrealistic future thinking were probed at -1 SD below the mean. The authors apologize for these errors in reporting and state that they do not change the scientific conclusions of the article in any way.

The correct legends appear below.

Figure 2 Positive future thinking moderates the association between defeat/entrapment and future (3-month) suicidal ideation. SDES, Short Defeat and Entrapment Scale; SIQ, Suicide Ideation Questionnaire. Greater and lower levels of positive future thinking were defined as +1 SD and -1 SD, respectively. The SIQ scale reflects values of the transformed variable, and not raw scores.

Figure 3 Degree of realistic positive future thinking moderates the association between defeat/entrapment and future (3-month) suicidal ideation. SDES, Short Defeat and Entrapment Scale; SIQ, Suicide Ideation Questionnaire. More vs. less realistic positive future thinking levels (i.e., less unrealistic vs. more unrealistic) were defined as $-1 \mathrm{SD}$ and $+1 \mathrm{SD}$, respectively. The SIQ scale reflects values of the transformed variable, and not raw scores.

A correction has been made to Procedure, Data Analyses, Paragraph 4. The corrected paragraph is shown below.

Thirdly, to test positive future thinking as a moderator, defeat/entrapment (i.e., SDES) and positive future thinking (i.e., FTT-Pos) variables were centered and multiplied to create an interaction term. Linear regressions were conducted with SDES, FTT-Pos, and (for analyses 
predicting follow-up SIQ) baseline SIQ entered in the first step. The interaction term was entered in the second step. Post-hoc probing analyses were conducted following guidance on testing moderation (Aiken and West, 1991; Holmbeck, 2002). Results of these post-hoc analyses were graphed at low ( -1 SD below the mean) and high ( +1 SD above the mean) levels of positive future thinking. Similar to Aims 1 and 2, additional post-hoc analyses explored baseline depressive symptoms (i.e., QIDS-SR) as a covariate in moderation models that significantly predicted suicidal ideation.

A correction has also been made to Results, Aim 3, Paragraph 3. The corrected paragraph is shown below.

We conducted additional post-hoc analyses addressing how positive future thinking may have been maladaptive in nature. Imagining many positive future events that are, for instance, detached from reality and unlikely to occur would presumably not be helpful. To determine how realistic adolescents' imagined positive events were, we assessed whether those events listed from baseline occurred over the next 3 months and calculated

\section{REFERENCES}

Aiken, L. S., and West, S. G. (1991). Multiple Regression: Testing and Interpreting Interactions. Newbury Park: Sage.

Holmbeck, G. N. (2002). Post-hoc probing of significant moderational and mediational effects in studies of pediatric populations. J. Pediatr. Psychol. 27, 87-96. doi: 10.1093/jpepsy/27.1.87

Publisher's Note: All claims expressed in this article are solely those of the authors and do not necessarily represent those of their affiliated organizations, or those of the publisher, the editors and the reviewers. Any product that may be evaluated in this article, or claim that may be made by its manufacturer, is not guaranteed or endorsed by the publisher. what proportion of them did not occur (i.e., unrealistic positive future thinking index). Indeed, the proportion of unrealistic positive future thinking moderated the association between defeat/entrapment and suicidal ideation 3 months later $(\beta=$ $0.17, p=0.03)$. We probed this result at higher $(+1 \mathrm{SD}$ above the mean) and lower ( -1 SD below the mean) levels of unrealistic future thinking (i.e., proportion of unrealized positive events) and found that defeat/entrapment predicted 3-month SIQ among those with less realistic future thinking (i.e., higher proportions of unrealized positive events; $\beta=$ 0.42, $p=0.004$ ), but not among those with more realistic future thinking (i.e., lower proportions of unrealized positive events; $\beta=0.12, p=0.38$; Figure 3 ). The interaction term between defeat/entrapment and unrealistic positive future thinking remained significant after controlling for depressive symptoms $(\beta=0.16, p=0.045)$.

The authors reiterate that these errors do not change the scientific conclusions of the article in any way. The original article has been updated.
Copyright (C) 2021 Pollak, Guzmán, Shin and Cha. This is an open-access article distributed under the terms of the Creative Commons Attribution License (CC BY). The use, distribution or reproduction in other forums is permitted, provided the original author(s) and the copyright owner(s) are credited and that the original publication in this journal is cited, in accordance with accepted academic practice. No use, distribution or reproduction is permitted which does not comply with these terms. 